with rhabdomyolysis, with or without exertional myalgia $(n=12)$, but no or little associated weakness; CK levels were markedly increased during episodes. Rhabdomyolysis was triggered by exercise and heat, viral infection and drugs. Familial RYR1 mutations were confirmed in relatives. (Dlamini N, Voermans NC, Lillis S, et al. Mutations in RYR1 are a common cause of exertional myalgia and rhabdomyolysis. Neuromuscul Disord 2013 Apr 28. pii: S0960-8966(13)00094-1 [Epub ahead of print]). (Response: Dr H Jungbluth, Children's Neurosciences Centre, St Thomas' Hospital, London SE1 7EH, UK. E-mail: Heinz.jungbluth@ghstt.nhs.uk).

COMMENT. Patients presenting with unexplained rhabdomyolysis and/or exertional myalgia and other family members should be tested for RYR1 mutations.

Malignant hyperthermia susceptibility of core myopathies. Due to their genetic linkage to mutations in the ryanodine receptor gene (RYR1), core myopathies (in particular, central core disease) carry a high risk of malignant hyperthermia susceptibility during anesthesia. (Brislin RP, Theroux MC. Paediatr Anaesth 2013 Apr 25 [Epub ahead of print]).

\title{
GLUCOCORTICOIDS FOR DUCHENNE MUSCULAR DYSTROPHY
}

Investigators at the Dubowitz Neuromuscular Centre, Great Ormond Street Hospital, and other centers in the UK, conducted a prospective longitudinal study across 17 neuromuscular centers in the UK of 360 boys aged 3-15 years with Duchenne muscular dystrophy who were treated with daily or intermittent (10 days on/10 days off) prednisolone for a mean duration of 4 years. The median loss of ambulation was 12 years in intermittent and 14.5 years in daily treatment; height restriction for intermittent versus daily regimen was $1.57(\mathrm{p}=0.13)$ and the median age for loss of ambulation did not differ. Boys on an intermittent regimen declined faster than those receiving daily treatment $(\mathrm{p}<0.001)$. Moderate to severe side effects were more common in the daily regimen, including Cushingoid features, hyperactive behavior and hypertension. Body mass index mean score was higher and height restriction was more severe in the daily regimen than in the intermittent regimen. (Ricotti V, Ridout DA, Scott E, et al. Long-term benefits and adverse effects of intermittent versus daily glucocorticoids in boys with Duchenne muscular dystrophy. J Neurol Neurosurg Psychiatry 2013 Jun;84(6):698-705). (Respond: Dr Francesco Muntoni, Dubowitz Neuromuscular Centre, UCL Institute of Child Health, 30 Guilford St., London WC1N 1EH, UK; E-Mail: f.muntoni@ucl.ac.uk).

COMMENT. Glucocorticoids are recommended in the international standards of care guideline for DMD and benefits are confirmed by Cochrane systematic reviews (Bushby K, et al. Neuromuscul Disord 2004 Sep;14(8-9):526-34). The most effective treatment postulated is prednisolone/prednisone or the equivalent deflazacort (Manzur AY, et al. Cochrane Database Syst Rev 2008 Jan 23;(1):CD003725). The intermittent and daily regimens are equally effective in gain of function until 6 years of age. After age 7 years, boys on an intermittent regimen decline more rapidly than those on daily therapy. Side effects are a greater problem with daily compared to intermittent therapy. 